\title{
Contribuições e desafios da Computação Desplugada: Um Mapeamento Sistemático
}

Emanuela Vitória Dias Morais, Instituto Federal de Educação Ciência e Tecnologia do

Sertão Pernambucano Campus Petrolina, vitoriadm98@gmail.com

Mayara Benício de B. Souza, Universidade de Pernambuco, mayarabenicio@gmail.com

Resumo: No presente artigo são apresentados os resultados de um mapeamento sistemático que contou com a análise de artigos sobre as contribuições e os desafios da computação desplugada, em dez repositórios nacionais e internacionais. Os resultados obtidos com a análise deste estudo destacam que grande parte das atividades desplugadas mapeadas tinham o objetivo de promover habilidades do pensamento computacional e, por isso, foi a contribuição mais citada nos artigos consultados. Por outro lado, o maior desafio encontrado foi com relação a eficácia das atividades desplugadas, desde a aplicação de forma inadequada ou isolada, e distante de experiências com as tecnologias digitais, ou a necessidade de trabalhar a computação desplugada junto com métodos online. Com a realização desta pesquisa pode-se concluir que a maior parte dos estudos primários mapeados eram voltados para alunos do ensino fundamental e, na sua maioria, abordavam habilidades do pensamento computacional e representação da informação.

Palavras-chave: computação desplugada, mapeamento sistemático, pensamento computacional.

\section{Contributions and challenges of Unplugged Computing: A Systematic Mapping}

Abstract: In the present article, we present the results of a systematic mapping that included the analysis of articles on the contributions and challenges of unplugged computing in ten national and international repositories. The results obtained with the analysis of this study point out that a large part of the mapped activities were designed to promote computational thinking skills and, therefore, was the most cited contribution in the articles consulted. On the other hand, the greatest challenge encountered was the effectiveness of the unplugged activities, from inadequately or isolated application, and away from experiences with digital technologies, or the need to work on unplugged computing together with online methods. With the accomplishment of this research one can conclude that the majority of the primary studies mapped were directed to elementary students and, for the most part, they approached computational thinking abilities and information representation.

Key-words: unplugged computing, systematic mapping, computational thinking.

\section{Introdução}

Atualmente, a computação tem estado cada vez mais presente no cotidiano das pessoas. No cenário educacional brasileiro, nos âmbitos da graduação e pós-graduação, já é comum a existência de cursos na área de Computação. Por outro lado, na educação básica ainda é necessário o desenvolvimento de habilidades computacionais (FRANÇA e AMARAL, 2013). 
O ensino de conceitos computacionais é geralmente confundido com aulas de informática que visam apenas utilizar a tecnologia como suporte para realizar tarefas relacionadas a outras ciências. Sobre isso, Scaico et al. (2012, p. 2) afirmam que:

(...) as escolas ainda estão em um estágio inicial desse processo [de ensino de computação] porque ensinar computação para crianças e adolescentes se confunde, por vezes, com as aulas de informática, que se referem à instruções voltadas para capacitar indivíduos para o manuseio de aplicativos de escritório, a edição gráfica e as ferramentas de gerenciamento de conteúdo web. (...) o modelo mental que os alunos desenvolvem sobre os sistemas computacionais ainda se molda a uma perspectiva de capacitar usuários de aplicativos.

Logo, não se pode limitar o ensino de Computação apenas ao manuseio do computador e suas aplicações básicas mas também usufruir das diversas ferramentas que podem contribuir para o processo de ensino-aprendizagem, até mesmo para resolver desafios reais através do pensamento computacional (PC). O PC é uma forma de resolver problemas, desenvolver sistemas e entender o comportamento humano através dos conceitos da ciência da computação (WING, 2006).

Assim, entende-se que para desenvolver habilidades do PC é preciso que o aluno torne-se capaz de pensar computacionalmente, por exemplo através da computação desplugada (BLIKSTEIN, 2008). A computação desplugada (CD) consiste na realização de atividades para o ensino de computação sem o uso de recursos tecnológicos, e é uma alternativa para executar atividades que estimulam o PC (MATOS, PAIVA e CORLETT, 2016).

Nesse contexto, o uso desta técnica democratiza o ensino dos conceitos computacionais, visto que não depende de recursos de alto custo para serem difundidos, e ainda desfaz o mito de que aprender computação é difícil, pois tem como base o estímulo do PC através de coisas do cotidiano (MANHÃES, GONÇALVES E CAFEZEIRO, 2017). Desta forma, este artigo pretende identificar através de um mapeamento sistemático quais são as contribuições e desafios da CD no ensino de Ciência da Computação.

\section{Metodologia}

O procedimento de coleta de dados deste trabalho diz respeito a uma pesquisa bibliográfica, realizada mediante um mapeamento sistemático de estudos sobre CD a fim de verificar suas contribuições e desafios quando aplicada na educação. Segundo Kitchenham e Charters (2007), mapeamento sistemático (MS) é uma ampla revisão de estudos em uma área de um tópico de pesquisa específico que visa identificar as evidências disponíveis sobre esse tópico. Para Kitchenham e Charters (2007) o MS é dividido em três fases: 1) Planejamento, no qual é determinado o objetivo e, definido e avaliado protocolo. 2) Condução, acontece a identificação e seleção dos estudos primários, e os dados são extraídos e sintetizados. 3) Publicação dos resultados, os resultados são descritos, divulgados e avaliados.

\subsection{Objetivos do mapeamento}

O estudo aqui apresentado teve por objetivo principal identificar um conjunto de atividades utilizadas na computação desplugada e as contribuições e desafios desta metodologia no ensino de computação em níveis de ensino diversos. Assim, a pesquisa partiu das seguintes questões: Q1) Quais os principais benefícios e vantagens do ensino de ciência da computação através de atividades desplugadas? Q2) Quais os principais desafios no ensino através da CD? Q3) Quais são as níveis de ensino onde a CD é mais 
utilizada, e o público alvo mais contemplado? Q4) Quais são os conteúdos mais explorados nas atividades de computação desplugada?

\subsection{Estratégia de busca}

Para responder às questões da pesquisa, foi utilizada a estratégia abaixo (ver Figura 1), a fim de determinar quais seriam os termos de busca.

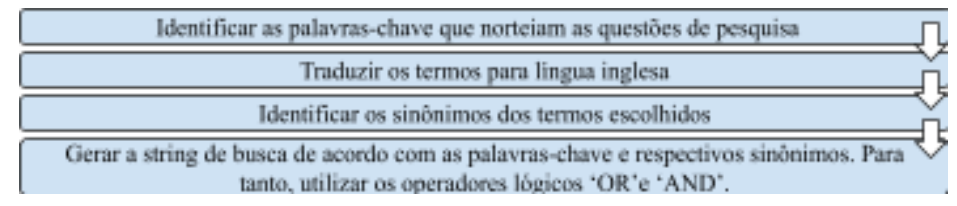

Figura 1: Estratégia para realização das buscas

Conforme as etapas definidas acima, as palavras-chave e respectivos sinônimos traduzidos para a língua inglesa utilizados na busca foram:

- Atividades, dinâmicas. Activities, Dynamics.

- Computação, ciência da computação. Computing, computer science;

- Desplugado, offline, lúdico, sem computador. Unplugged, offline, ludic, no computer.

De acordo com as palavras-chave encontradas, foram geradas as strings de busca, como mostra a tabela abaixo:

Tabela 1: Strings de busca utilizada no mapeamento sistemático

\begin{tabular}{|l|}
\hline \multicolumn{1}{|c|}{ Strings de busca } \\
\hline $\begin{array}{l}\text { ("unplugged computing" OR "unplugged science computer" OR "computing ludic" OR "unplugged dynamics" } \\
\text { OR "unplugged activities" OR "computing no computer" OR "offline computing") }\end{array}$ \\
\hline $\begin{array}{l}\text { ("computação desplugada" OR "ciência da computação desplugada" OR "computação lúdica" OR "dinâmica } \\
\text { desplugada" OR "atividade desplugada" OR "computação sem computador" OR "computação offline") }\end{array}$ \\
\hline
\end{tabular}

Fonte: elaborado pela autora (2018).

A string em língua inglesa foi utilizada nos mecanismos de busca: IEEEXplore, ACM e Springer Link. A string em português foi utilizada nos demais repositórios: BDTD, RENOTE, WEI, WIE, SBIE, CBIE e Periódicos CAPES.

\subsection{Critérios de inclusão e exclusão}

Foram incluídos os estudos completos disponíveis exclusivamente em meio eletrônico em repositórios web de relevância científico-acadêmico, foram excluídos os estudos que se enquadraram em algum dos seguintes critérios: i) Repetidos: se o trabalho for reproduzido novamente em fontes de busca diferentes; ii) Duplicados: trabalhos com estudos semelhantes. Neste caso foi apenas o trabalho mais recente ou com informações mais completas; iii) Irrelevantes para o objetivo da pesquisa; iv) Não apresentavam como foco principal o ensino de Computação; v) Não eram estudos primários; vi) Com textos, conteúdo ou resultados incompletos.

\section{Resultados e Discussão}

A busca resultou em 366 artigos: 8 do IEEEXplore, 87 do ACM, 167 do Springer Link, 73 do BDTD, 1 da RENOTE, 4 do WEI, 10 do WIE, 6 do SBIE, 8 do CBIE e 2 dos Periódicos CAPES. Após a primeira seleção (leitura dos títulos e palavras-chaves) dos trabalhos, resultaram 64 estudos potencialmente relevantes para a pesquisa. A segunda seleção (leitura da introdução e conclusão) identificou 19 estudos que foram 
incluídos como estudos primários e utilizados na extração dos dados. O formulário de aprovação dos estudos primários está disponível no link: https://tinyurl.com/y2lyt88k.

\subsection{Evolução temporal dos estudos}

A busca por estudos primários foi indiferente quanto ao ano de suas publicações, sendo estes estudos encontrados entre 2012 e 2018. Nestes 6 anos houve uma evolução que demonstra a popularização da CD ao longo dos anos. A disposição da quantidade de estudos por ano de publicação pode ser verificada na Figura 2 abaixo.

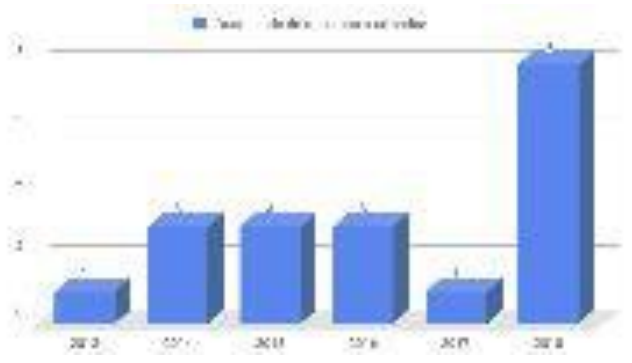

Figura 2: Número de estudos primários ao longo dos anos

De acordo com a Figura 2, os anos com a menor quantidade de publicações foram 2012 e 2017, com apenas 1 trabalho publicado. Seguindo com 3 trabalhos nos anos 2014, 2015 e 2016. O ano que apresentou o maior número de publicações foi 2018. Neste ano houve 8 publicações. Tal observação sugere uma tendência recente no desenvolvimento de pesquisas sobre de computação desplugada, principalmente por ser uma temática difundida há poucos anos.

\subsection{Distribuição dos estudos por país}

Os estudos primários mapeados estão distribuídos em 5 países, dos quais o Brasil tem a maior representatividade, com 15 estudos selecionados. Os outros países foram: Peru, Alemanha, Suíça e Reino do Bahrein, e contam com 1 publicação cada. A figura 3 apresenta esta distribuição de publicações por países.

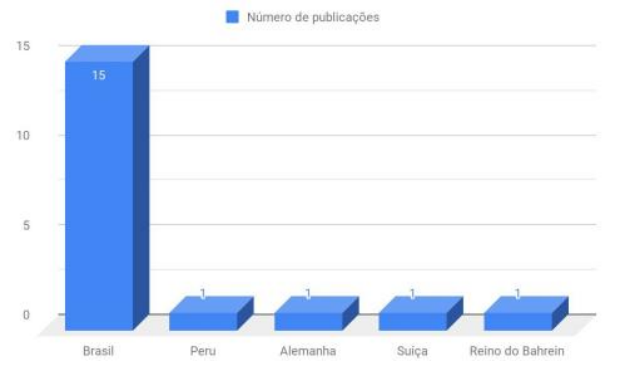

Figura 3: Representatividade dos estudos primários por país

Como a maior representatividade dos estudos primários com relação ao país de publicação foi o Brasil, é apresentada na Figura 4, a representatividade desses estudos, considerando a região geográfica onde as pesquisas relatadas nos estudos primários foram desenvolvidas.

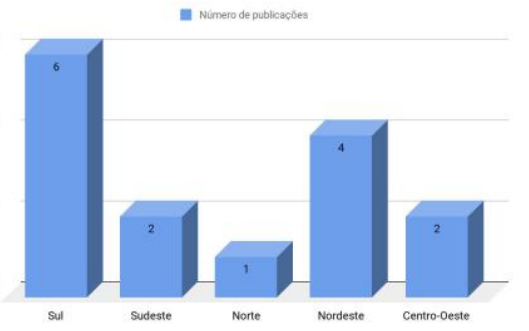

Figura 4: Representatividade dos estudos publicados no Brasil, considerando o local de desenvolvimento da pesquisa, por região geográfica 
De acordo com a Figura 4, a Região Sul tem o maior número de estudos primários sobre CD mapeados. Em seguida, a Região Nordeste, com 4, Sudeste e Centro-Oeste, com 2 estudos. A maior representatividade da região Sul demonstra um nítido interesse pela $\mathrm{CD}$ em suas escolas, em detrimento da região Norte que apresentou apenas 1 estudo primário.

\subsection{Evidências Brutas do Mapeamento Sistemático}

Com os estudos primários selecionados nesta pesquisa foi possível obter evidências de atividades, contribuições e desafios da computação desplugada. Essas evidências brutas, ou seja, os dados resultantes do MS estão descritos na Figura 5 que representa para cada EP, a quantidade de atividades desplugadas extraídas, as contribuições e desafios na sua utilização.

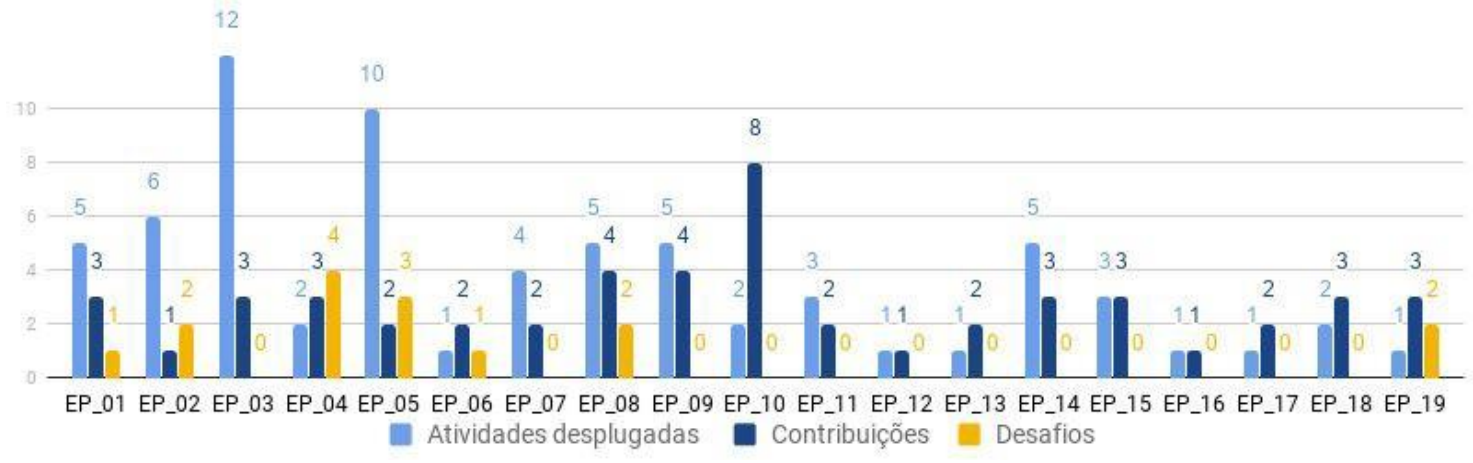

Figura 5: Resultado das evidências brutas obtidas dos estudos primários

A Figura 5 apresenta evidências de 70 atividades desplugadas, 52 contribuições e 15 desafios advindos da sua utilização distribuídas entre os 19 estudos primários mapeados. $\mathrm{O}$ estudo primário que apresentou o maior número de atividades de $\mathrm{CD}$ foi o EP_03, com 12 atividades. O EP_10 foi o mais representativo no que diz respeito às contribuições destacadas, 8 no total. Com relação aos desafios, o EP_04 obteve maior representatividade, com 4 desafios relatados. As categorias a seguir apresentam as evidências brutas pormenorizadas e as questões de pesquisa delineadas neste trabalho juntamente com as respostas adquiridas pelos estudos primários.

\subsection{Contribuições no ensino através da computação desplugada}

As contribuições mapeadas foram relacionadas as atividades aplicadas nas experiências relatadas, ou relatos dos autores sobre a abordagem desplugada. Dos 19 estudos analisados, em todos eles foram encontradas pelo menos uma contribuição. A Tabela 2 apresenta as contribuições mapeadas, com seu respectivo identificador e o estudos primários dos quais foram extraídas.

Tabela 2: Contribuições mapeadas nos estudos primários

\begin{tabular}{|l|l|l|}
\hline C_ID & \multicolumn{1}{|c|}{ Contribuição } & \multicolumn{1}{|c|}{ EPs } \\
\hline C_01 & Simplificação do conceitos de programação & EP_01 \\
\hline C_02 & Mudança da opinião sobre ciência da computação (CS) & EP_01, EP_10 \\
\hline C_03 & Compreensão e domínio da CS e/ou da programação & EP_01, EP_10 \\
\hline C_04 & Envolvente para manter a atenção dos alunos na aula & EP_02 \\
\hline C_05 & Preencher lacunas de conhecimento dos professores sobre o PC & EP_03 \\
\hline C_06 & Capacitar professores para ensinar computação & EP_03, EP_04 \\
\hline C_07 & Introduzir conceitos de ciência da computação para professores adultos & EP_03 \\
\hline C_08 & Promover habilidades do PC & EP_03, EP_05, EP_06, EP_09, \\
& & EP_10, EP_13, EP_14, EP_15, \\
\hline
\end{tabular}




\begin{tabular}{|c|c|c|}
\hline & & EP_19 \\
\hline C_09 & Motivação/interesse/envolvimento & EP_04, EP_13, EP_14, EP_18 \\
\hline C_10 & Ajudar os alunos com computação plugada (conectada) & EP_04 \\
\hline C_11 & Ensino em locais sem infraestrutura adequada & EP_05, EP_09, EP_19 \\
\hline C_12 & Promover o ensino de Matemática e temas transversais & EP_06 \\
\hline C_13 & Descontração, colaboração, trabalho em equipe & EP_07, EP_18 \\
\hline C_14 & Promover a compreensão da lógica de programação & EP_07, EP_18 \\
\hline C_15 & Contato com os conceitos e fundamentos de tecnologias & EP_08, EP_10 \\
\hline C_16 & Quebrar paradigmas quanto às modalidades de ensino de computação & EP_08 \\
\hline C_17 & Diversão (aprender brincando) & EP_08 \\
\hline C_18 & $\begin{array}{l}\text { Facilidade em assimilar o conteúdo e completar com sucesso as atividades, } \\
\text { exercícios e desafios }\end{array}$ & EP_08 \\
\hline C_19 & Facilita o tratamento de problemas & EP_09 \\
\hline C_20 & Promover o ensino de algoritmos, sem computadores & EP_09 \\
\hline C_21 & Aplicação dos conhecimentos da informática de forma lúdica & EP_10 \\
\hline C_22 & Descoberta de novos conhecimentos & EP_10 \\
\hline C_23 & Utilização da tecnologia em prol da educação & EP_10 \\
\hline C_24 & Aulas atrativas & EP_10 \\
\hline C_25 & Aprendizado em cenário colaborativo & EP_11 \\
\hline C_26 & Participação ativa do sujeito no processo de aprendizagem & EP_11, EP_13 \\
\hline C_27 & Introduzir conceitos de coleta, análise e representação de dados & EP_12 \\
\hline C_28 & Desenvolvimento do raciocínio lógico/matemático & EP_14, EP_15, EP_17, EP_19 \\
\hline C_29 & Melhoria no rendimento escolar & EP_15 \\
\hline C_30 & Motivar o ensino de programação paralela & EP_16 \\
\hline C_31 & Inclusão Digital de alunos e professores & EP_19 \\
\hline
\end{tabular}

A Tabela 2 apresenta 31 contribuições distintas sobre a CD, distribuídas entre os 19 estudos primários mapeados. A contribuição de maior relevância foi promover o PC, citada em 9 estudos (47,3\%). Outro tópico relevante foi o destaque para a motivação/interesse/envolvimento dos alunos frente às atividades de $\mathrm{CD}, 5$ estudos deixam claro essa evidência.

E ainda 4 estudos relatam contribuições com relação ao desenvolvimento do raciocínio lógico/matemático. Essas contribuições destacam a importância da CD no ensino de Ciência da Computação, principalmente para desenvolver habilidades do raciocínio computacional, lógico e matemático, além de ser importante para motivar os alunos durante às aulas.

\subsection{Desafios no ensino através da computação desplugada}

Os desafios podem ser relacionados às atividades aplicadas nas experiências relatadas, ou relatos dos autores sobre a abordagem desplugada. Dos 19 estudos analisados em apenas 8 deles foram encontrados algum desafio. Os desafios mapeados com seu respectivo identificador e o mecanismo do qual foi extraído estão descritos na Tabela 3 abaixo.

Tabela 3: Desafios mapeados nos estudos primários

\begin{tabular}{|l|l|c|}
\hline D_ID & \multicolumn{1}{|c|}{ Desafio } & EPs \\
\hline D_01 & $\begin{array}{l}\text { Foco: geralmente tem enfoque em atividades de nível macro, e ignoram conceitos de programação } \\
\text { de nível micro. }\end{array}$ & EP_01 \\
\hline D_02 & Avaliação: determinar o que os alunos estão aprendendo & EP_02 \\
\hline D_03 & Estruturação e conteúdo: as atividades precisam de mais estrutura e conteúdo & EP_02 \\
\hline
\end{tabular}




\begin{tabular}{|l|l|l|}
\hline D_04 & Atual e conectado: precisa estar ligado à tecnologia atual e não deve ser usado apenas isoladamente & EP_04 \\
\hline D_05 & $\begin{array}{l}\text { Solução de problemas: garantir que os problemas apresentados realmente têm uma solução bem } \\
\text { definida }\end{array}$ & EP_04 \\
\hline D_06 & $\begin{array}{l}\text { Conhecimento: desenvolver ou ampliar as atividades podem exigir conceitos não básicos de ciência } \\
\text { da computação garantir que os exemplos são simples ou ilustram adequadamente as ideias-chave }\end{array}$ & EP_04 \\
\hline D_07 & Eficácia: se usado de forma inadequada, pode ser ineficaz ou causar danos & EP_04 \\
\hline D_08 & Eficácia e Conexão: possível questionar sua eficácia quando utilizada isoladamente & EP_05 \\
\hline D_09 & $\begin{array}{l}\text { Completude: não atende todos os fundamentos da Computação ou não proporciona uma prática } \\
\text { plena. }\end{array}$ & EP_05 \\
\hline D_10 & $\begin{array}{l}\text { Eficácia e conexão: podem não ser tão eficazes quanto esperado, por manter os alunos distantes de } \\
\text { experiências com as tecnologias digitais }\end{array}$ & EP_05 \\
\hline D_11 & $\begin{array}{l}\text { Elaboração: as atividades precisam ser bem elaboradas para não causar complicações no } \\
\text { entendimento por parte dos alunos. }\end{array}$ & EP_06 \\
\hline D_12 & Componente curricular: inexistência de uma disciplina & EP_08 \\
\hline D_13 & $\begin{array}{l}\text { Espaço: não exige laboratório, mas demanda salas de aula com espaço para as dinâmicas e } \\
\text { atividades em grupo. }\end{array}$ & EP_08 \\
\hline D_14 & Preparação: professores não se sentem preparados para trabalhar com o tema & EP_19 \\
\hline D_15 & Vontade: professores não desejam trabalhar com assuntos ligados à computação. & EP_19 \\
\hline
\end{tabular}

Na Tabela 3 pode-se observar 15 desafios mapeados nos estudos primários. $\mathrm{O}$ principal deles está relacionado a eficácia das atividades de CD (EP_04 e EP_05), desde a aplicação de forma inadequada ou isolada e distante de experiências com as tecnologias digitais. Além disso, alguns estudos destacam que é necessário trabalhar a computação desplugada junto com métodos online.

\subsection{Níveis de ensino e público-alvo}

As evidências encontradas concentram-se em Educação Infantil, Ensino Fundamental, Ensino Médio, Ensino Superior, Ensino Técnico e Capacitação, conforme apresentada na Figura 6.

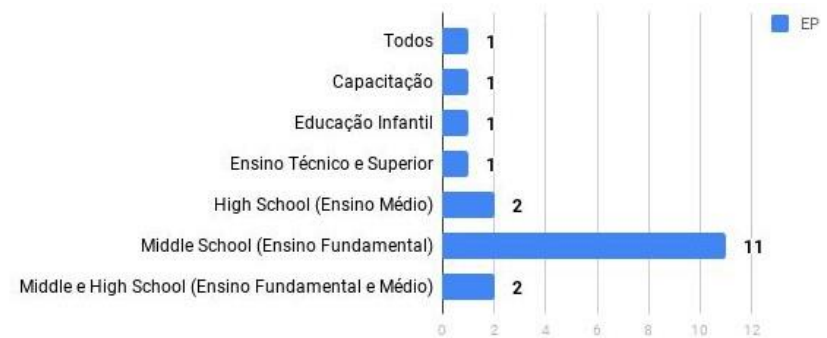

Figura 6: Distribuição dos estudos primários de acordo com o nível de ensino

De acordo com os dados apresentados, pode-se observar que a maioria dos estudos retratam experiências voltados somente para o ensino fundamental, com representação em 11 estudos primários (EP_02, EP_05, EP_06, EP_08, EP_09, EP_11, EP_12, EP_14, EP_15, EP_17, EP_18), o qual corresponde a 57,9\% do total. Tais resultados sugerem que a CD tem sido mais difundida na educação básica.

O público alvo foi dividido de acordo com a função dos indivíduos - alunos ou professores - para quem as atividades desplugadas são direcionadas. A Figura 7 apresenta uma visão geral da quantidade de estudos para cada público.

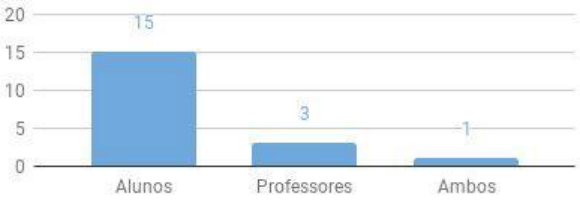


Figura 7: Distribuição dos estudos primários de acordo com o público-alvo

Conforme os dados apresentados, pode-se observar que o público-alvo predominante nos estudos são os alunos, com representação em 15 estudos primários, que corresponde a 78,9\% do total. Apenas 3 estudos $(15,7 \%)$ tem como público-alvo os professores, e 1 estudo voltado tanto para professores quanto alunos. Estes resultados apontam uma necessidade de trabalhos utilizando computação desplugada direcionados a professores, a fim de propiciar o conhecimento da metodologia e, consequentemente, maior utilização.

\subsection{Conteúdos}

Os conteúdos foram classificados da forma como estavam descritos nos EPs mapeados e estão detalhados na Tabela 4 que ilustra todos os temas encontrados nas atividades desplugadas, o respectivo identificador e o mecanismo do qual foi extraído.

Tabela 4: Distribuição dos estudos primários por conteúdo

\begin{tabular}{|c|c|c|}
\hline CT_ID & Conteúdo & EPs \\
\hline CT_01 & Programação - funções & EP_01, EP_15 \\
\hline CT_02 & Programação - variáveis locais e globais & EP_01, EP_03, EP_09 \\
\hline CT_03 & Programação - listas & EP_01 \\
\hline CT_04 & Programação - Estrutura condicional & EP_01, EP_15, EP_09, EP_15 \\
\hline CT_05 & Programação - instrução & EP_01 \\
\hline CT_06 & Programação - Estrutura de repetição & EP_01, EP_09, EP_15 \\
\hline CT_07 & Autômatos de Estado Finito & EP_02 \\
\hline CT_08 & Representação da informação & EP_02, EP_06, EP_08, EP_11 \\
\hline CT_09 & Criptografia & EP_02, EP_10, EP_15 \\
\hline CT_10 & Detecção de erros & EP_02, EP_10 \\
\hline CT_11 & Árvores de abrangência mínima & EP_02 \\
\hline CT_12 & Pesquisa & EP_02 \\
\hline CT_13 & Programação - atribuição & EP_03 \\
\hline $\mathrm{CT} \_14$ & Programação - declarações & EP_03 \\
\hline CT_15 & Programação - fluxo de controle & EP_03 \\
\hline CT_16 & Interação humano-computador (IHC ) & EP_03 \\
\hline CT_17 & Pensamento Computacional & EP_03, EP_04, EP_05, EP_07, EP_09, EP_15, EP_17 \\
\hline CT_18 & Redes de ordenação & EP_04 \\
\hline CT_19 & Teoria dos grafos & EP_04, EP_14 \\
\hline CT_20 & Algoritmos & EP_04, EP_16, EP_19 \\
\hline $\mathrm{CT} \_21$ & Resolução de problemas & EP_07 \\
\hline CT_22 & Funcionamento do computador & EP_07 \\
\hline CT_23 & Tratamento da informação & EP_07, EP_12 \\
\hline CT_24 & Estrutura Sequencial & EP_09, EP_15, EP_19 \\
\hline $\mathrm{CT} \_25$ & Banco de Dados & EP_13 \\
\hline CT_26 & Lógica de Programação - Posicionamento & EP_14 \\
\hline CT_27 & Lógica de Programação - Ordenação & EP_14, EP_18 \\
\hline CT_28 & Lógica de Programação - Agrupamento & EP_14 \\
\hline CT_29 & Depuração & EP_19 \\
\hline
\end{tabular}

No quadro 3.2 pode-se observar 29 conteúdos abordados nas atividades desplugadas mapeadas nos estudos primários. De acordo com os dados apresentados, o conteúdo que apresenta a maior representatividade é o PC, trabalhado em atividades 
desplugadas de 7 estudos primários (EP_03, EP_04, EP_05, EP_07, EP_09, EP_15, EP_17). Seguido de representação da informação e estrutura condicional, presentes em 4 estudos cada um destes.

\section{CONCLUSÃO E TRABALHOS FUTUROS}

O presente trabalho apresentou os resultados de um mapeamento sistemático da literatura, com o objetivo de identificar um conjunto de atividades utilizadas na $\mathrm{CD}$ e as contribuições e desafios desta metodologia no ensino de computação. Foram elaboradas quatro perguntas de pesquisa para guiar o estudo e após a análise de 366 trabalhos, 19 estudos primários foram selecionados para responder às questões da pesquisa.

Com relação a representatividade dos estudos por mecanismos de busca, apesar de 45,6\% dos estudos retornados serem do mecanismo de busca Springer Link, sua representatividade no total dos estudos primários foi apenas de 5\%. Ao contrário do que ocorreu no mecanismo do Workshop de Informática na Escola (WIE) que representou apenas $2,7 \%$ dos estudos retornados, mas obteve a maior representatividade nos estudos primários, com $31,6 \%$ do total.

Foi possível verificar 31 contribuições diferentes distribuídas entre os 19 estudos primários o que possibilitou inferir sobre a importância da utilização da CD para o ensino de Ciência da Computação. A principal contribuição mapeada diz respeito a promoção das habilidades do Pensamento Computacional. Esta contribuição foi descrita em $47,3 \%$ dos estudos primários selecionados.

Quanto aos desafios obtidos com o uso de atividades desplugadas para o ensino de ciência da computação a extração dos dados permitiu observar que o principal desafio na utilização da $\mathrm{CD}$ foi a dúvida quanto a sua eficácia, tanto com relação a aplicação de forma inadequada ou isolada e a distância de experiências que envolvam tecnologia. Outros estudos destacam a necessidade de trabalhar a CD junto com métodos online. Isso permite considerar esses desafios como fatores cruciais na elaboração e aplicação de atividades desplugadas.

Com relação a evolução do número de estudos sobre CD, o ano de 2018 apresenta o maior número de publicações. Devido a atualidade do tema compreende-se que haja mais publicações nos anos mais recentes. Os 19 estudos primários estão divididos entre os anos 2012 e 2018. O ano de 2012 tem a menor representatividade no número de publicações, com apenas 1 publicação, e 2018 a maior, com 8 publicações.

Os estudos primários foram classificados de acordo com o público-alvo e o nível de ensino. De acordo com os dados apresentados, 78,9\% dos estudos foram realizados com somente com alunos. O nível de ensino com a maior representação foi o ensino fundamental, com 11 estudos (57,9\%). Isso possibilita inferir que a CD tem sido mais difundida entre alunos do ensino fundamental com a finalidade principal de promover habilidades do PC.

E, por fim, com relação ao conteúdo abordado nas atividades desplugadas mapeadas foram identificados 29 conteúdos distintos distribuídos entre os 19 estudos primários. O conteúdo com a maior representatividade foi o pensamento computacional o qual esteve presente em atividades de 7 estudos.

Dessa forma, este estudo permitiu obter um conhecimento sobre um conjunto de atividades utilizadas na CD e contribuir para a estruturação de forma sistemática das informações acerca das contribuições e desafios no ensino através da $C D$, servindo como base para o desenvolvimento de trabalhos futuros na área, bem como para o desenvolvimento de atividades desplugadas para o ensino. 
Como trabalhos futuros sugere-se realizar uma análise de qualidade das atividades desplugadas selecionadas nos estudos a fim de verificar a facilidade de uso para o público alvo e, se estas são eficazes para o ensino dos conteúdos propostos. Além disso sugere-se refazer este estudo dentro de 5 anos visando observar se houve alguma mudança nas tendências levantadas no presente texto.

\section{REFERÊNCIAS}

BELL, T.; WHITTEN, I.; FELLOWS, M. Computer Science Unplugged. Ensinando Ciência da Computação sem o uso do computador. Tradução de Luciano Porto Barreto. 2011.

BLIKSTEIN, P. O Pensamento Computacional e a Reinvenção do Computador na Educação. $2008 . \quad$ Disponível em: http://www.blikstein.com/paulo/documents/online/ol_pensamento_computacional.html. Acesso em: 13 mai. 2018.

FRANÇA, R. S.; AMARAL, H. J. C. Ensino de Computação na Educação Básica no Brasil: Um Mapeamento Sistemático. In: Anais do XXXIII Congresso da Sociedade Brasileira de Computação, Maceió, 2013. Disponível em http://www.lbd.dcc.ufmg.br/colecoes/wei/2013/009.pdf. Acesso em 15 mai. 2018.

LOBO, R. A Evasão No Ensino Superior Brasileiro - Novos Dados. Estadão. 07 out. 2017. Educação. $\quad$ Disponível em https://educacao.estadao.com.br/blogs/roberto-lobo/497-2/. Acesso em: 24 jun. 2018. KITCHENHAM, B.; CHARTERS, S.. Guidelines for performing Systematic Literature Reviews in Software Engineering. Keele University. Durham, UK, 2007. Disponível em: https://userpages.uni-koblenz.de/ laemmel/esecourse/slides/slr.pdf. Acesso em 13 mai. 2018.

MANHÃES, T.S.; GONÇALVES, F. S.; CAFEZEIRO, I. Computação Desplugada e Educada. In: Anais VII Esocite.br/tecsoc. ISSN 1808-8716. p. 1-25. 2017. Disponível em esocite2017.com.br/anais/beta/.../gt/34/esocite2017_gt34_isabelCafezeiro.pdf Acesso em 16 mai. 2018.

MATOS, E., PAIVA, F; CORLETT, E. Novas atividades de computação desplugada para promoção de integração curricular na escola. In: RAABE A. L. A. et al. Educação Criativa: Multiplicando experiências para a aprendizagem. Recife: Pipa Comunicação, 2016. p. 206-249.

SCAICO, P. D., et al. Um Relato de Experiências de Estagiários da Licenciatura em Computação com o Ensino de Computação para Crianças. Revista Novas Tecnologias na Educação. V. 10, no 3, 2012. p. 2. ISSN 1679-1916. Disponível em http://seer.ufrgs.br/index.php/renote/article/view/36377/23471. Acesso em 13 mai. 2018.

VIEIRA, A.; PASSOS, O.; BARRETO, R. Um Relato de Experiência do Uso da Técnica Computação Desplugada. In: Anais do XXI WEI, p. 670-679. 2013. Disponível em: http://www.lbd.dcc.ufmg.br/colecoes/wei/2013/0031.pdf. Acesso em 16 mai. 2018.

WING, J. M. Computational thinking. Communications of the ACM, v. 49, n. 3, p. 33, 2006. Disponível em https://www.cs.cmu.edu/ 15110-s13/Wing06-ct.pdf. Acesso em 13 mai. 2018. 